McGill Law Journal

Revue de droit de McGill

\title{
Hugo Cyr, Canadian Federalism and Treaty Powers: Organic Constitutionalism at Work, New York, PIE Peter Lang, 2009
}

\section{François Chevrette}

Volume 58, numéro 3, march 2013

URI : https://id.erudit.org/iderudit/1018395ar

DOI : https://doi.org/10.7202/1018395ar

Aller au sommaire du numéro

Éditeur(s)

McGill Law Journal / Revue de droit de McGill

ISSN

0024-9041 (imprimé)

1920-6356 (numérique)

Découvrir la revue

Citer ce compte rendu

Chevrette, F. (2013). Compte rendu de [Hugo Cyr, Canadian Federalism and Treaty Powers: Organic Constitutionalism at Work, New York, PIE Peter Lang, 2009]. McGill Law Journal / Revue de droit de McGill, 58(3), 775-791.

https://doi.org/10.7202/1018395ar d'utilisation que vous pouvez consulter en ligne.

https://apropos.erudit.org/fr/usagers/politique-dutilisation/ 
McGill Law Journal Revue de droit de McGill

\author{
RECENSION CRITIQUE \\ Hugo Cyr, Canadian Federalism and Treaty Powers: Organic Consti- \\ tutionalism at Work, New York, PIE Peter Lang, 2009.
}

\title{
François Chevrette
}

La question de savoir si les provinces canadiennes disposent d'un pouvoir constitutionnel de conclure des traités dans leurs champs de compétence en est une qui a été assez abondamment débattue, à la fois en doctrine et dans les débats d'affaires publiques ${ }^{1}$; mais il faut dire d'emblée que le présent apport du professeur Hugo Cyr est ce qu'il y a de plus exhaustif et de plus approfondi dans tout ce qui a été écrit à ce sujet à ce jour.

Canadian Federalism and Treaty Powers: Organic Constitutionalism at $W_{o r k}{ }^{2}$ est une thèse de doctorat, légèrement remaniée, que ce dernier a soutenue à l'Université de Montréal en juin 2007 et dont un membre du jury, constitutionnaliste émérite et évaluateur externe, a dit en séance qu'il s'agissait de la meilleure thèse de doctorat qu'il avait lue dans toute sa carrière. Outre le fait que l'ouvrage débute de la façon la plus vivante qui soit ${ }^{3}$, ce qui le distingue de cet aspect aride que l'on prête, à tort et à raison, à bien des dissertations académiques, la thèse n'en est pas une que par le nom - bien des thèses n'étant que de bonnes analyses dépourvues de prise de position -, mais aussi par le fond, puisque son auteur défend bec et ongles l'idée que les provinces canadiennes ont le pouvoir de conclure des traités dans les champs de compétence qui sont les leurs et que

* Professeur titulaire, Faculté de droit, Université de Montréal. Texte posthume.

(C) François Chevrette 2013

Citation: (2013) 58:3 McGill LJ 775 Référence : (2013) 58 : 3 RD McGill 775

1 Hugo Cyr, Canadian Federalism and Treaty Powers: Organic Constitutionalism at Work, New York, PIE Peter Lang, 2009, aux notes infrapaginales 40, 41, 54 (au sujet des États-Unis) et 55 (journaux) pour d'abondantes bibliographies.

2 Bien choisi, ce titre de portée générale s'explique par le fait que l'auteur se penche à la fois sur le pouvoir de conclure des traités et sur celui de les mettre en œuvre législative, l'accent étant surtout mis sur le premier de ces deux pouvoirs.

3 L'auteur nous rappelle, en nous indiquant même le temps qu'il faisait ce 12 avril 1965, le discours que le ministre Paul Gérin-Lajoie adressait au corps consulaire de Montréal et qui visait à démontrer que le Québec disposait de l'extension externe de ses compétences, en d'autres mots qu'il avait le pouvoir de signer et ratifier des traités dans ses champs de compétence. Voir supra note 1 à la p 13 et $\mathrm{s}$. 
leur pouvoir, bien reconnu, de les mettre en œuvre législativement, ne devrait d'aucune façon être remis en question.

Comme il se doit, je ferai d'abord un rapide survol de l'ouvrage pour ensuite m'appliquer à mettre en relief ce qui constitue, selon moi, ses cinq caractéristiques les plus marquantes.

Je ne reviens pas sur sa stimulante entrée en matière ${ }^{4}$, tenant cependant à ajouter que si les mots cadre d'analyse et méthodologie sonnent parfois un peu creux à mes oreilles, la dichotomie théorique que Hugo Cyr nous présente en introduction entre une vision volontariste (on pense aux ruptures et recommencements des États-Unis et de la France en matière constitutionnelle) et une vision organique (on pense à une progression plutôt qu'à un acte plus ou moins instantané de volonté, le tout à l'anglaise et à la canadienne) me réconcilie avec l'idée d'un cadre théorique, cadre qui, dans le cas présent, structure tout le reste du texte en même temps qu'il pourrait se lire et se méditer isolément. J'y reviendrai.

Très simplement et très logiquement, l'étude se divise en deux parties. Dans la première, que je chapeauterais du titre "Un héritage non concluant ", l'auteur, pour arriver à ce constat, se livre à une analyse approfondie du fameux arrêt sur les Conventions de travail, tant en Cour suprême du Canada qu'au Comité judiciaire du Conseil privés, et de la jurisprudence un peu tortueuse qui l'a précédé6. La deuxième partie est à triple volet. Elle comporte d'abord un volet négatif, où l'auteur ne se contente pas de réfuter tous les arguments légaux traditionnellement avancés à l'appui d'une compétence fédérale exclusive en matière de conclusion de traités, mais présente plusieurs arguments fonctionnels (ou de policy) à l'encontre d'une telle compétence. Le volet positif, quant à lui, s'attarde d'abord à décrire avec minutie l'implication des provinces canadiennes dans les relations internationales. Cela fait, l'auteur s'interroge sur la légalité de ces implications, tant au regard du droit constitutionnel que du droit international. C'est l'occasion pour lui d'avoir recours, un peu à la façon dont il l'a fait sous le volet négatif, à des arguments fonctionnels et même à certains appels à la théorie des jeux, le tout au soutien du pouvoir des provinces de conclure des traités dans leurs champs de compétence. Le troisième volet de cette deuxième partie pourra, en première analyse, surprendre puisqu'il n'y est plus question de la conclusion des traités,

4 Ibid.

5 References re Weekly Rest in Industrial Undertaking Act, Minimum Wages Act and Limitations of Hours of Work Act, [1936] RCS. 461, 3 DLR 673; Canada (PG) v Ontario (PG), [1937] AC 326, 1 DLR 673 (PC) [Ontario (PG) avec renvois aux AC].

6 Supra note 1 aux pp 61-67 (cette étrange histoire, un peu oubliée aujourd'hui, est fort bien relatée par l'auteur). 
mais bien de leur mise en œuvre législative. Il est bien établi, depuis l'arrêt sur les Conventions de travail du Comité judiciaire ${ }^{7}$, que la mise en œuvre législative des traités portant sur des sujets de compétence provinciale relève des provinces. Mais comme certains juristes souhaiteraient que la Cour suprême modifie cela au profit du Parlement fédéral, Hugo Cyr a cru approprié de démontrer avec force, premièrement, l'absence de fondement légal à un tel revirement, deuxièmement, ses inimaginables conséquences sur l'équilibre du fédéralisme, et troisièmement, l'extrême difficulté d'opérer pareille révolution même en passant par la voie de l'amendement constitutionnel.

Voilà pour l'ossature de ce livre. Ajoutons-y maintenant la chair et les muscles! J'espère pouvoir donner une idée de la profondeur et de l'originalité de cette importante réflexion en centrant mes commentaires autour de ses cinq caractéristiques selon moi les plus marquantes: l'exhaustivité de l'analyse (I), l'originalité de la méthode (II), un solide encadrement théorique (III), une bonne place à l'analyse fonctionnelle (IV) et, enfin, une fructueuse érudition (V).

\section{L'exhaustivité de l'analyse}

Hugo Cyr détient son double diplôme de premier cycle en droit de la Faculté de droit de l'Université McGill, auquel se sont ajoutés une maîtrise en droit de Yale et un doctorat en droit de l'Université de Montréal. Qu'on me permette de dire que son livre est une bonne illustration de cette magnifique pensée d'Euripide que la Faculté de droit de McGill a eu le génie de faire graver dans la pierre, à droite de l'entrée principale de la Bibliothèque de droit et qu'on a fort exactement traduite en anglais par "To leave no stone unturned" (la citation en grec est là aussi, de même que sa traduction française). L'exhaustivité de l'analyse de l'auteur frappe dès la première partie, que j'ai titrée précédemment par l'expression «Un héritage non concluant ". De l'important avis consultatif du Comité judiciaire sur les Conventions de travail, on dit à peu près toujours la même chose, à savoir qu'on y a reconnu la compétence des provinces sur la mise en œuvre législative des traités ayant un objet provincial. Mais ici, ce sont aussi les argumentations des gouvernements fédéral, de l'Ontario, du Nouveau-Brunswick et de la Colombie-Britannique devant le Comité judiciaire qui sont décortiquées, comme le sont les opinions des six juges ayant participé au jugement de la Cour suprême ainsi, bien entendu, que l'avis de Lord Atkin pour le Comité judiciaire. Rien donc n'est négligé pour

$7 \quad$ Ontario $(P G)$, supra note 5. 
cette cruciale remise en contexte, et c'est ce que j'appelle du vrai scholarship ${ }^{8}$.

De cette minutieuse analyse, retenons trois choses. D'abord la curieuse pirouette de l'Ontario qui, après avoir mis de l'avant tous les arguments favorisant les provinces, concède que la théorie des dimensions nationales, dérivée du paragraphe introductif de l'article 91 de la Loi constitutionnelle de $1867^{9}$, habilite le Parlement fédéral à mettre en œuvre législativement les traités portant sur des sujets de compétence provinciale! Plus importante est ensuite la démonstration, par Hugo Cyr, que le jugement de la Cour suprême, au contraire d'une opinion assez largement répandue, n'est aucunement concluant quant à la compétence fédérale exclusive de conclure des traités, même ceux portant sur des sujets provinciaux. Pour l'essentiel, la démonstration consiste à faire voir que les arguments sur la base desquels trois des six juges ayant participé au jugement (le juge en chef Duff et les juges Davis et Kerwin) ont conclu à une compétence fédérale générale sur la conclusion et la mise en œuvre des traités, compétences indissociables l'une de l'autre selon eux, ont tous été rejetés sur appel au Comité judiciaire, l'opinion de deux autres juges (les juges Crocket et Cannon) favorables à une compétence fédérale générale sur la conclusion des traités n'étant qu'obiter dicta puisque sur la question en litige - celle de savoir si les provinces peuvent mettre en œuvre législative des traités à objets provinciaux - ils ont répondu affirmativement et que cela suffit. Ne poussons pas plus loin le résumé de l'analyse de l'auteur, si ce n'est pour souligner que celle qu'il fait de l'avis de la Cour suprême n'est jamais dissociée de l'avis que devait rendre le Comité judiciaire un an plus tard - ce qui est juridiquement irréprochable ${ }^{10}$ - et que sur certains points assez secondaires, il me semble forcer quelque peu la note ${ }^{11}$.

8 C'est Montaigne qui écrivait, à propos des sources de seconde main : «Il y a plus affaire à interpréter les interprétations qu'à interpréter les choses, et plus de livres sur les livres que sur autre subject : nous ne faisons que nous entregloser " (Abel-François Villemain, dir, Les essais de Montaigne (1588 et variantes de 1595), t 7, Paris, Jouaust, 1889, ch 8 à la p 9). La remarque, pertinente pour bien des études juridiques, ne l'est absolument pas pour le présent ouvrage, où les sources sont invariablement de première main.

9 (R-U), 30 \& 31 Vict, c3, art 91, reproduite dans LRC 1985, ann II, $\mathrm{n}^{\circ} 5$ [Loi constitutionnelle de 1867].

10 Supra note 1 à la p 85. L'auteur n'a jamais entendu analyser l'avis de la Cour suprême isolément et pour le seul plaisir de le faire mais pour vérifier s'il constitue un " binding precedent ", ce qui supposerait que le Comité judiciaire ne l'ait pas infirmé.

11 En Cour suprême, le juge Rinfret a reconnu à l'autorité fédérale le pouvoir de conclure et de mettre en œuvre des traités à objets provinciaux, à condition d'avoir l'accord des provinces avant leur conclusion. Le juge Cannon opte pour la compétence provinciale de 
Arrivons au troisième élément de cette mise en contexte. Hugo Cyr veut ici démontrer que l'avis du Comité judiciaire n'appuie aucunement la thèse d'une compétence fédérale exclusive en matière de conclusion de traités et qu'il constitue plutôt une infirmation implicite (" a silent overruling ${ }^{12}$ ) de l'avis de la Cour suprême à ce sujet. Or, l'auteur a l'honnêteté de reproduire ${ }^{13}$ un extrait de l'avis du Comité judiciaire où ce dernier, en insistant, d'une part, sur la compétence provinciale de mise en œuvre législative des traités à objets provinciaux et en écartant, d'autre part, l'idée d'un parallélisme parfait entre le pouvoir de conclure et le pouvoir de mettre en œuvre, peut sembler laisser entendre que le pouvoir de conclure, lui, n'est pas divisible et qu'il appartient en totalité au gouvernement central. Mais comme aucun argument n'est soumis par Lord Atkin à l'appui de cette position, par ailleurs plus qu'incertaine, l'auteur reporte son analyse à la deuxième partie de son livre où il démontrera que, puisque selon lui aucun pareil argument n'existe, ce ne peut être ce que Lord Atkin a voulu dire. Mais avant d'en arriver là, il fait une analyse soigneuse de la façon dont Lord Atkin établit la compétence provinciale exclusive de mise en œuvre législative des traités à objets provinciaux pour nous rappeler, à la fin de ce développement, la métaphore si souvent citée du savant Lord: "While the ship of statement sails on larger ventures and into foreign waters she still retains the watertight compartments which are essential part of her original structure $»^{14}$. Développement qui garde toute son utilité puisque, comme on l'a rappelé plus haut, d'aucuns voudraient que soit mise de côté cette position du Comité judiciaire, remise en question dont, comme on l'a vu et le reverra, l'auteur se présente comme un farouche adversaire.

À la vérité, l'exhaustivité de l'analyse est partout présente dans cet ouvrage, mais je me permets ici d'attirer l'attention sur deux développements dont je n'aurai pas l'occasion de reparler plus loin et qui cadrent particulièrement bien avec la présente rubrique de mon appréciation. Il s'agit, premièrement, de celui où l'auteur rejette les arguments juridiques souvent invoqués à l'appui d'un pouvoir fédéral exclusif de conclure des

mise en œuvre et, en obiter, estime l'autorité fédérale compétente sur la conclusion, à condition de consulter les provinces au sujet des traités "affecting the provinces". Hugo Cyr, supra note 1 aux pp 86-89, prétend que le terme "affecting" est employé ici par opposition à l'expression "relative to " - une distinction classique il est vrai en droit constitutionnel - et que dans l'opinion du juge Cannon il n'y a simple consultation que pour les traités touchant très incidemment ("affecting") les pouvoirs des provinces. On peut se demander si le juge Cannon avait vraiment cette distinction à l'esprit.

13 Supra note 1 à la p 84 .

14 Ontario $(P G)$, supra note 6 à la p 354. 
traités ${ }^{15}$. Deuxièmement, il s'agit de celui où il rejette pareillement les arguments militant en faveur de la compétence du Parlement fédéral de mise en œuvre législative des traités à objets provinciaux ${ }^{16}$. Évoquons très brièvement ces deux développements, étant entendu que certaines composantes d'entre eux seront examinées plus loin, soit parce qu'elles illustrent particulièrement bien la méthode de l'auteur (II), soit parce qu'elles présentent un fort intérêt théorique (III) ou qu'elles sont de caractère fonctionnel (IV).

Quant au premier de ces deux développements, celui relatif aux arguments juridiques souvent avancés à l'appui d'un pouvoir fédéral exclusif de conclure des traités, c'est probablement la partie la plus complexe de l'ouvrage, dont les conclusions, sans être toutes incontestables, sont toutes fortement vraisemblables et dont on peut dire que rien n'y est ignoré et que toutes les difficultés y sont abordées de front. Ni les Lettres patentes de 1947 du Souverain britannique ni les prérogatives de la Couronne n'appuient, selon l'auteur, la thèse d'un pouvoir fédéral exclusif de conclure des traités. Les premières auraient opéré dévolution de matières ne concernant que le gouvernement central et n'auraient rien à voir avec le pouvoir de conclure des traités - sauf peut-être les plus solennels, les traités "Head of State", guère plus en vogue aujourd'hui puisqu'Ottawa avait acquis ce pouvoir bien avant 1947. Quant aux prérogatives, le raisonnement de l'auteur, par moment un peu tortueux, est cependant à juste titre centré sur le principe indiscutable que la prérogative est en dépendance du pouvoir législatif et non l'inverse. Si, comme c'est le cas, le Parlement fédéral n'a pas compétence pour mettre en œuvre les traités portant sur les matières provinciales, la prérogative de les conclure n'a rien à quoi se raccrocher et il n'est pas soutenable, selon lui et avec raison à mon avis, qu'un transfert de prérogatives découlant de la divisibilité de la Couronne - une prérogative allant toujours en diminuant, elle ne peut être conceptualisée que comme un tel transfert et non comme un accroissement au profit des gouvernements fédéral et provinciaux puisse avoir donné lieu à de nouveaux pouvoirs législatifs. Voilà qui va à contre-courant de l'opinion de plusieurs juristes, opinion souvent marquée par la redite, le stéréotype et la superficialité.

Parce que peu contestable, passons vite sur l'idée que le pouvoir fédéral de conclure des traités ne peut être le fruit d'une convention constitutionnelle ou d'un simple usage et insistons plutôt sur le sort que l'auteur réserve à cette curieuse notion de "souveraineté extérieure", qui n'est

15 Supra note 1 aux pp 104-58.

16 Ibid aux pp 218-57. 
pas " a term of art " ${ }^{17}$ dans notre droit constitutionnel et qui, dans le contexte où elle fut explicitement ${ }^{18}$ ou implicitement ${ }^{19}$ employée, doit être interprétée, selon Hugo Cyr, comme visant la question spécifique à l'étude, à savoir l'absence de compétence et de droit de propriété des provinces sur la mer territoriale et le plateau continental. "These Supreme Court opinions are not enough, in my view, to support the propositions that (a) there is only one international legal personality in Canada and (b) the federal government is its representative ${ }^{20}$. Ce ne serait rien de plus que la réitération de l'absence de compétence extraterritoriale des provinces. Or, l'approfondissement théorique de la notion de personnalité internationale est à ce point au cœur de l'ouvrage pour que j’y revienne plus loin sous la dernière rubrique « Une fructueuse érudition » (V).

S'agissant du développement où se trouve fermement réfutée toute prétention fédérale à la mise en œuvre législative des traités à objets provinciaux, ce qui retient l'attention, en plus de l'idée que l'article 132 de la Loi constitutionnelle de 1867 est désuet et ne peut que le demeurer, est la démonstration que pareille prétention ne peut trouver appui ni dans le paragraphe introductif de l'article 91 - ce qu'on appelle parfois le pouvoir général du Parlement fédéral — ni dans l'étonnante proposition, souvent avancée, qu'un traité échappe par nature aux matières locales provinciales et aux compétences strictement territoriales des provinces. Sur tous ces sujets, l'ouvrage emporte tout simplement la conviction, tant il paraît peu discutable, d'une part, que la mise en œuvre des traités n'a ni l'unicité ni la spécificité pour faire l'objet des pouvoirs résiduels fédéraux et que, d'autre part, c'est précisément sur le territoire de l'État contractant que l'effet d'un traité se fait sentir et non à l'extérieur.

\section{L'originalité de la méthode}

Ce livre, je l'ai annoncé plus haut, se distingue aussi par l'originalité de la méthode. Qu'est-ce à dire? Un peu tautologiquement sans doute de ma part, elle consiste à opter pour une méthode et à s'en tenir à elle quand vient le temps des analyses spécifiques et des exemples concrets. Or, dans le présent cas, le cœur de cette méthode réside à la fois dans un

17 Ibid à la $\mathrm{p} 121$.

18 Renvoi relatif au plateau continental de Terre-Neuve, [1984] 1 RCS 86 à la p 116, 5 DLR (4) 385 .

19 Avis sur les droits miniers sous-marins, [1967] RCS 792, 65 DLR (2e) 353.

20 Supra note 1 à la $\mathrm{p}$ 117. En plus des deux avis cités aux deux notes précédentes, l'auteur procède à une analyse très soigneuse de l'opinion majoritaire quant à la légalité de la démarche fédérale dans le Renvoi: Résolution pour modifier la Constitution ([1981] 1 RCS 753, 125 DLR (3e) 1), le tout pour conclure à l'incohérence et à l'intérêt purement historique de ce qui y est dit sur la personnalité internationale du Canada. 
souci de cohérence conceptuelle ${ }^{21}$ et de fidélité à la perspective organique canadienne $^{22}$. En d'autres termes, sont à éviter autant le conservatisme inadapté des idées, des principes et des structures que les emprunts, importations et bouleversements méritant le même qualificatif. Quand je relis ces pages auxquelles je viens de faire référence - de même que celles sur les communautés existentielles et les régimes fonctionnels ${ }^{23}$ auxquelles je reviendrai plus loin, toutes pages qui mériteraient de figurer dans n'importe quelle anthologie de théorie, voire de philosophie du droit — j’ai bien du mal à me dissocier de mon collègue émérite qui, examinateur externe sur le jury de cette thèse, en a fait l'appréciation que j'ai rappelée plus haut. Mais, venons-en aux applications de cette méthode, que l'auteur définit comme centrée sur ce qui est "possible within the bounds defined by current constitutional norms and their possible organic extension ${ }^{24}$. J'en retiens deux.

Je choisis comme première illustration de cette méthode la façon dont l'auteur affiche une complète fin de non-recevoir à un éventuel transfert au Parlement fédéral de la compétence des provinces de mise en œuvre législative des traités à objets provinciaux. C'est sous ma troisième rubrique, celle consacrée à l'encadrement théorique de l'ouvrage, que je rappellerai les caractéristiques qu'avec bien d'autres, il est vrai, l'auteur décèle dans les traités modernes, remarquant avec raison que ceux-ci engagent leurs signataires autant, sinon plus, sur des questions internes qu'internationales. Rappelons ici simplement qu'aux fins de sa présente démonstration, il prend comme exemples les deux grands pactes de l'ONU, celui sur les droits civils et politiques ${ }^{25}$ et celui sur les droits économiques, sociaux et culturels ${ }^{26}$. De l'un et de l'autre, il extrait la substance, très largement de compétence provinciale, surtout dans le cas du deuxième, et brosse le tableau d'un fédéralisme canadien méconnaissable et qui serait l'antithèse absolue de ses deux postulats méthodologiques évoqués plus haut s'il devait arriver que le Parlement fédéral hérite de la compétence de mise en œuvre législative des traités, même ceux à objets provinciaux, et s'il décidait de l'exercer en l'espèce. Ce développement ${ }^{27}-$ qui a au fond pour but de montrer l'exceptionnelle étendue d'une compé-

21 Supra note 1 aux pp 51-58 (« Conceptual Maintenance»).

22 Ibid aux pp 33-51.

23 Ibid aux pp 172-81.

24 Ibid à la $\mathrm{p} 97$.

25 Pacte international relatif aux droits civils et politiques, 19 décembre 1966, 999 RTNU 171, RT Can $n^{\circ} 47$.

26 Pacte international relatif aux droits économiques, sociaux et culturels, 16 décembre 1966, 993 RTNU 3, RT Can 1976 n $^{\circ} 46$.

27 Ibid aux pp 233-35. 
tence fédérale générale sur la mise en œuvre des traités, ce qui en fait, si l'on peut dire, une compétence vraiment pas comme les autres - coupe simplement le souffle du lecteur et je l'ai pour ma part beaucoup apprécié, prêt que je suis à répondre aux critiques qu'on peut lui faire tant qu'elles sont, selon moi, sans fondement.

En effet, d'aucuns diront probablement que ces exemples sont exagérés, et mal choisis puisque ces deux traités, sans doute précisément en raison de leur ampleur, n'ont pas fait l'objet d'une mise en ouvre législative systématique, ni par le Parlement fédéral ni par les législatures. Mais qu'importe. Le constitutionnaliste, quand il analyse la question de savoir si une compétence législative existe ou pas, n'a pas à se soucier de son éventuel exercice, modéré ou abusif ${ }^{28}$. Le pouvoir existe ou il n'existe pas et, dans l'hypothèse où il existe, il est parfaitement approprié d'en faire voir toutes les conséquences. C'est le propre d'une vraie thèse que d'acculer au pied du mur les défenseurs de la thèse opposée. Hugo Cyr est ici un ardent et dangereux adversaire!

Un autre exemple où sont mises en relief l'importance des deux postulats méthodologiques de l'auteur et la difficulté pratique d'y déroger en l'espèce est fourni par la formule de modification constitutionnelle ${ }^{29}$ dans l'hypothèse où l'on déciderait d'en faire usage pour enlever aux provinces leur compétence sur la mise en œuvre des traités à objets provinciaux et pour la transférer au Parlement fédéral. Voici ce que Hugo Cyr nous rappelle à ce sujet ${ }^{30}$ : pareil amendement tomberait certes sous la formule générale de l'article 38(1), formule dite des $2 / 3$ - 50\%, avec toutefois l'exigence d'une majorité renforcée au Parlement et dans les législatures provinciales puisque, comme le prévoit l'article 38(2), il s'agirait d'une modification dérogatoire à la compétence des provinces. Mais ce n'est pas tout. Tombant sous l'article 38(2), cette modification tomberait aussi et par le fait même sous l'article 38(3), de sorte qu'une province pourrait s'y opposer et maintenir pour elle le statu quo. De par la généralité même de son objet, la modification impliquerait à coup sûr transfert de compétence sur des questions éducatives et culturelles, auquel cas le Canada devrait, conformément à l'article 40, fournir compensation à la ou aux provinces

28 Sauf bien entendu si un exercice abusif était de nature à faire disparaître la compétence, comme on en a décidé à propos de l'impôt direct provincial (Loi constitutionnelle de 1867, supra note 10, art 92(2)) quand il frappe avec excès les banques et qu'il perd sa nature fiscale pour devenir une mesure bancaire, de compétence fédérale exclusive ( $L o i$ constitutionnelle de 1867, supra note 10, art 91(15)). Voir en particulier Alberta (PG) c Canada (PG) (1938), [1939] AC 117, [1938] 4 DLR 433 (PC). Une telle limitation est inexistante pour la prétendue compétence à l'étude ici.

29 Loi constitutionnelle de 1982, art 38-48, constituant l'annexe B de la Loi de 1982 sur le Canada (R-U), 1982, c 11.

30 Supra note 1 aux pp 252-57. 
tenant au statu quo s'il décidait d'exercer sa nouvelle compétence sur les matières en question. Ajoutons à cela que l'auteur, étant du ferme avis que les provinces ont le pouvoir de conclure des traités dans leurs champs de compétence, nous montre que le transfert serait en ce cas singulièrement encore plus compliqué vu que des prérogatives de la Couronne seraient probablement en cause.

Un processus si plein d'embûches donne à penser qu'il ne serait pas respectueux d'une méthode centrée sur la cohérence conceptuelle et la dimension organique du fédéralisme canadien. L'auteur en déduit aussi que si d'aventure c'était le pouvoir judiciaire qui faisait le revirement, ce ne serait rien de moins qu'un coup d'État ${ }^{31}$.

\section{Un solide encadrement théorique}

Un solide encadrement théorique : telle est, selon moi, la troisième caractéristique de cet ouvrage. Et cet encadrement est particulièrement frappant à propos surtout de la conception du fédéralisme prônée par l'auteur, associée à sa forte remise en question du concept de souveraineté, à propos aussi de la nature et de l'objet des relations internationales et des traités internationaux de notre époque. La conception que l'auteur expose de la notion de personnalité internationale ${ }^{32}$ mériterait aussi de figurer sous la présente rubrique. J'en reporterai toutefois le survol sous la dernière, "Une fructueuse érudition » (V).

$\mathrm{Au}$ sujet du fédéralisme, revenons d'abord brièvement sur la distinction, sur laquelle l'auteur élabore en début d'ouvrage ${ }^{33}$, entre un constitutionnalisme volontariste et un constitutionnalisme organique. Très riche et fortement documentée, cette réflexion se prête mal à un simple résumé. J'en retiens uniquement ici qu'il s'agit d'une typologie et que le Canada, "strange beast " ${ }^{34}$, participe des deux traditions, avec toutefois une très nette prépondérance pour la deuxième en particulier en matière de relations internationales et de traités internationaux. "[I]ndependance did not result in a momentuous reconstruction of the Canadian constitutional order $»^{35}$, " the constitutional imperatives relative to treaty powers in Canada are entirely the product of the Organic growth of the Canadian constitution ${ }^{36}$ et "[t]o reverse the current position would not simply mean ad-

\footnotetext{
$31 \quad$ Ibid à la p 257.

32 Ibid aux pp 136-59, 181-86.

33 Ibid aux pp 33-51.

$34 \quad$ Ibid à la $\mathrm{p} 43$.

35 Ibid à la $\mathrm{p} 41$.

36 Ibid à la $\mathrm{p} 49$.
} 
justing a few constitutional strings here and there; it would mean a radical redrawing of the Canadian federation $»^{37}$. N'ayant pas abusé jusqu'ici des extraits de l'ouvrage, je me suis permis, vu leur importance, de reproduire ceux qui précèdent! Ils illustrent bien la façon dont l'auteur appréhende le pouvoir des provinces canadiennes de conclure des traités dans leurs champs de compétence et le maintien de leur pouvoir de les mettre en œuvre législativement.

La perspective organique du fédéralisme canadien n'est toutefois pas la seule sur laquelle l'auteur trouve appui. Font aussi partie de ce tableau théorique, au meilleur sens du mot, le principe de subsidiarité et la distinction, majeure et d'intérêt - sans exagération - universel, entre les communautés existentielles et les régimes fonctionnels ${ }^{38}$. De quoi s'agit-il?

Autoriser le gouvernement fédéral à conclure des traités à objets provinciaux et, le cas échéant, autoriser le Parlement fédéral à les mettre en œuvre législativement, c'est présenter le principe de subsidiarité la tête en bas et les pieds en l'air. Alors même que la subsidiarité commande que les décisions étatiques soient prises en principe par l'autorité la plus proche du citoyen, sauf si une autorité plus centrale peut les prendre beaucoup plus efficacement (on pense par exemple au combat contre les pollutions transfrontières), on donnerait ici au pouvoir central la responsabilité de s'occuper de ce que la Constitution même reconnaît déjà clairement comme de la compétence des provinces. Le monde à l'envers quoi! À moins bien sûr qu'on adopte le point de vue anachronique selon lequel le fait pour un domaine de responsabilité publique de faire l'objet d'un traité en change radicalement la nature!

$\mathrm{Au}$ cœur de la théorie du fédéralisme que Hugo Cyr met de l'avant dans son livre se situe évidemment aussi la distinction, évoquée plus haut, entre les communautés existentielles et les régimes fonctionnels. Le plaidoyer - j'emploie le mot à dessein, tant de monde restant à convaincre de cette conception - met en relief une conception du fédéralisme pleinement ouverte à ce que j'appellerais la pluralité et la variabilité des allégeances, telle région ou telle communauté pouvant primordialement s'identifier au pouvoir central, existentiellement pour reprendre la terminologie de l'auteur, telle autre et avec une intensité comparable à un pouvoir plus localisé, la première conservant une allégeance moins affective et plus simplement pratique et fonctionnelle à ce dernier, et inversement pour la deuxième.

$37 \quad$ Ibid à la $\mathrm{p} 50$.

38 Ibid aux pp 172-81. 
Bien évidemment les exemples canadiens et québécois viennent immédiatement à l'esprit. Il est clair en effet que pour une majorité de Québécois la communauté existentielle se centre autour de l'État provincial, le gouvernement central étant plutôt vu comme le régime fonctionnel, et inversement pour d'autres provinces, sous réserve de bien des nuances. Mais la théorisation déborde le cas du Canada et plus encore la question des traités, objet du présent ouvrage. Faisant grand état de la multiplicité des acteurs sur la scène internationale moderne, avec lesquels l'on entretient un éventail de relations, allant d'existentielles à fonctionnelles en passant par toutes les nuances intermédiaires, cette théorisation aboutit inévitablement à une véhémente remise en question du concept d'État souverain. En représentant l'État comme une personne humaine, constitué comme elle et assumant les mêmes rôles ${ }^{39}$, pareil anthropomorphisme d'un autre âge est difficilement compatible avec une pluralité de communautés existentielles. La communauté existentielle, selon cette vision, c'est l'État dit souverain et rien d'autre; ce qui conduit l'auteur, centrant cette fois son attention sur le Canada, à qualifier de souverainistes autant ceux pour qui le pouvoir central est la seule et vraie communauté existentielle, entouré de dix régimes fonctionnels provinciaux, que les souverainistes québécois qui n'en ont que pour la communauté existentielle québécoise. Le fédéralisme suppose au contraire, comme je le notais plus haut, pluralité et variabilité des allégeances. Pour en revenir à la conclusion des traités, nous sommes ici aux antipodes du slogan bien connu "One Country, One Voice ". Fidèle à la riche conceptualisation que je viens de résumer bien imparfaitement, Hugo Cyr suggère plutôt : " Many Ways to "One Voice" ${ }^{40}$. S'ensuit une intéressante analyse des modalités possibles de coopération interprovinciale visant à ce que les provinces harmonisent entre elles leurs relations extérieures et où sont cités à la barre l'exemple de la Belgique, celui du Conseil des ministres de l'Éducation du Canada et certains mécanismes institutionnels et décisionnels de l'Union européenne.

Vu le sujet de son livre, l'auteur ne pouvait évidemment éviter de nous rappeler la transformation profonde de l'objet des relations internationales et des traités internationaux à l'époque où nous vivons. Cet autre axe théorique de l'ouvrage, moins original que celui sur le fédéralisme, nous rappelle quand même opportunément que si les traités d'alliance et les relations proprement interétatiques ne sont aucunement exclus du domaine, ce sont avant tout les problèmes traditionnellement domestiques qui incitent aujourd'hui les acteurs internationaux à se concerter. Le traité n'est plus, du moins primordialement, cette forme de bouclier 
protégeant l'appareil de gouvernance interne contre l'étranger; il est devenu un des instruments mêmes de cette gouvernance. Et la réduction du formalisme contractuel ainsi que la multiplication des " acteurs" internationaux sont la conséquence de cet élargissement du traité à ces nombreux domaines nouveaux ${ }^{41}$ : contrats, développement économique, famille, adoption, droits de la personne et combien d'autres. D'où le lien à faire entre ces domaines de la coopération internationale et la liste des compétences que la Constitution canadienne octroie aux provinces. Ils sont si nombreux à en relever qu'au nom de la "Conceptual Maintenance " le pouvoir des provinces de conclure des traités dans leurs champs de compétence s'impose à la fois conceptuellement et fonctionnellement. Ce qui m'amène à la quatrième caractéristique, et non la moindre, de ce livre, à savoir la place importante faite à l'analyse fonctionnelle ("policy approach »).

\section{Une place importante à l'analyse fonctionnelle}

Le droit constitutionnel canadien ne fait pas, à mon avis, une assez large place à l'analyse fonctionnelle, encore que ce ne soit pas ici le lieu pour tenter d'expliquer cette carence. Or Hugo Cyr y a, avec raison, largement recours, à la fois pour démontrer qu'il n'est pas souhaitable de donner au gouvernement central le pouvoir de conclure des traités dans les secteurs de compétence provinciale et qu'il est au contraire souhaitable de reconnaître ce pouvoir aux provinces. Examinons brièvement et successivement cette double démonstration.

Commençons par le premier volet ${ }^{42}$. Un ministère fédéral des Affaires étrangères ayant à la fois l'expertise et la responsabilité de toutes les questions de compétence fédérale présentant une dimension internationale est aujourd'hui une vue de l'esprit en raison de la variété et de la technicité de ces questions et de l'intérêt qu'elles présentent pour nombre d'autres ministères. En l'état actuel des choses, les Affaires étrangères relevant d'au moins quatre ministères ${ }^{43}$, on est en droit de conclure, avec l'auteur, que si le ministère des Affaires étrangères proprement dit n'a pas l'expertise pour embrasser l'ensemble des domaines fédéraux à incidence internationale, "we can easily imagine the difficulties it faces in re-

41 Ibid aux pp 200-01.

42 Ibid aux pp 165-81.

43 Le ministère des Affaires étrangères, le ministère du Commerce international, le ministère de la Défense nationale et le ministère de la Coopération internationale. 
lation to provincial matters ${ }^{44}$. Compétence des provinces de conclure des traités s'entend donc ici dans le double sens de pouvoir et d'expertise!

$\mathrm{Au}$ titre des arguments fonctionnels militant contre l'autorité du gouvernement fédéral de conclure des traités en matières provinciales se retrouve aussi l'impératif de l'imputabilité démocratique. Sur ce sujet, l'auteur pose plusieurs questions dont je ne retiens ici que celle de savoir si Ottawa est le mieux placé pour arbitrer des intérêts provinciaux divergents et pour décider d'aller ou de ne pas aller de l'avant avec un traité international à objet provincial dans l'hypothèse où certaines provinces $\mathrm{y}$ sont favorables et d'autres pas. Ottawa fait cet arbitrage et ne peut se refuser à le faire quand des questions fédérales sont en jeu; et c'est à l'ensemble de l'électorat fédéral qu'il devra en répondre. Mais dans le cas sous étude, il y a comme une dissociation entre le décideur et les provinces dont les intérêts sont inadéquatement représentés. Personne ne pourrait dire que c'est là de la bien bonne démocratie!

L'argumentation fonctionnelle, je l'ai déjà dit, occupe une place importante dans cet ouvrage à la fois dans son volet négatif que je viens simplement d'évoquer, mais aussi dans son volet positif, celui-ci visant à montrer que le pouvoir des provinces de conclure des traités internationaux est éminemment souhaitable d'un point de vue fonctionnel.

À la différence des simples problèmes de coordination où le facteur temps n'intervient pas - la conduite automobile à droite ou à gauche en est un bon exemple -, la coopération, dont le fameux dilemme du prisonnier démontre le caractère avantageux, s'échelonne dans le temps et la chaîne des engagements peut se briser n'importe quand si certaines conditions ne sont pas remplies. Il faut donc une entente à laquelle les parties vouent leur loyauté et leur réputation; il faut que le nombre de jeux ou d'échanges ne soit pas déterminé à l'avance, à défaut de quoi chaque partie sera incitée à cesser de collaborer sachant la partie terminée et la riposte impossible; il faut enfin et surtout que ladite entente soit vraiment génératrice d'obligations juridiques, condition pour que sa violation porte atteinte à la loyauté et à la réputation de la partie en défaut. Et l'auteur d'ajouter, faisant référence à la nécessité que bien des ententes, au-delà des engagements, doivent comporter des structures et des mécanismes institutionnels: "I am not talking about cases where parties would not be willing to cooperate without an agreement, but rather about cases where certain forms of cooperation would not even be possible, even in principle, without an agreement ${ }^{45}$.

44 Supra, note 1 à la p 170.

45 Ibid à la p 208. 
Est-ce trop dire que ce développement ${ }^{46}$ suggère qu'il est futile et irréaliste de mettre en doute le caractère obligatoire des accords provinciaux, à la fois internationaux et interprovinciaux? Et ce développement devient d'autant plus concret qu'il est précédé par une longue et minutieuse description des implications des provinces canadiennes dans le champ des relations internationales ${ }^{47}$. Ainsi l'on voit bien de quoi il s'agit.

Il me faut insister ici sur le fait que si j'ai mis l'accent sur les analyses fonctionnelles de l'auteur, ce n'était nullement dans l'intention de reléguer au second plan ses analyses plus classiquement juridiques dont j'ai tenté de donner une idée sous ma première rubrique "L'exhaustivité de l'analyse " (I).

\section{Une fructueuse érudition}

Au-delà de la qualité et de l'exhaustivité de la documentation, ce en quoi le présent ouvrage mérite une claire mention d'excellence, il faut encore être capable de s'en servir et de conforter la ou les thèses que l'on défend par des appuis et des parallèles tout à la fois nationaux, étrangers, comparatifs, théoriques et historiques. Érudition que je qualifie de fructueuse en ce qu'elle n'est pas un étalage gratuit de connaissances, mais une construction intellectuelle complexe qui nourrit les démonstrations auxquelles l'on veut procéder.

Or ici à nouveau le présent ouvrage n'est rien de moins qu'excellent, ce que je me contenterai d'illustrer par un seul exemple, qui est toutefois au cœur même de la thèse : la notion de personnalité internationale ${ }^{48}$.

Ce que Hugo Cyr dénonce avec force, c'est la confusion longtemps entretenue - et encore, quoique de moins en moins - entre souveraineté étatique et personnalité internationale, confusion qui conduit à conclure que seul l'État souverain possède la personnalité internationale, lui seul pouvant par conséquent conclure des traités. Or, la dénonciation de l'auteur prend appui sur des sources nombreuses, variées et attentivement analysées, tout en précisant bien que la question à l'étude en est une de droit constitutionnel et non de droit international public et tout en poussant même la précaution jusqu'à l'analyse des modes de réception de ce dernier en droit interne. Voilà qui n'interdit aucunement toutefois de s'inspirer en l'espèce du droit international public et des expériences étrangères, ce à quoi Hugo Cyr s'applique, et avec profit.

\footnotetext{
46 Ibid aux pp 201-10.

47 Ibid aux pp 186-96.

48 Ibid aux pp 136-59, 181-86.
} 
Après l'étude de certaines grandes Conventions internationales, de certains prononcés de la Cour internationale de justice et de l'opinion des internationalistes les plus éminents, il s'en remet ensuite aux exemples, eux aussi multiples, pour conclure que la personnalité internationale signifie " the capacity to be a bearer of rights and obligations, [the bearers being] all sorts of legal entities having all sorts of different rights, powers and obligations [...] [I]ndividuals, universities, municipalities, nongovernmental organisations, multinational corporations, intergovernmental organisations, etc. all benefit from having restricted forms of international status $»^{49}$.

La démonstration est instructive à la fois, si je puis dire, dans l'espace et dans le temps. Dans l'espace parce qu'à l'aide de sources toujours de première main, elle met en évidence la personnalité internationale d'entités fédérées, régionales ou communautaires dans les cas, et j'en passe, de l'Argentine, de l'Autriche, de l'Allemagne, de la Belgique, des cantons suisses et, pour un passé pas si lointain, de la Biélorussie et de l'Ukraine. Dans le temps parce que l'auteur a le souci, avec l'appui d'une impressionnante documentation, de remonter jusqu'aux origines historiques de la notion de "persona jure gentium" pour nous apprendre que cette notion fut développée, non pas au profit de ce qui allait devenir l'État souverain moderne, mais bien pour faire une place, dans l'univers diplomatique de l'époque, à de nouvelles entités politiques émergentes et à faire en sorte que le Pape et l'Empereur partagent dorénavant cet univers avec ces dernières, le tout sur la base de leurs missions particulières. Et l'auteur d'établir, en conclusion à cette analyse, un intéressant parallèle entre cette forme d'arbitrage historique et ce qui s'est passé entre Ottawa et Québec en 1971 lorsque les deux capitales, délaissant cette chape de fer qu'est la souveraineté, ont convenu que le Québec ait statut de participant, avec représentation distincte, au sein de ce qu'on connaît aujourd'hui sous l'appellation d'Agence intergouvernementale de la Francophonie ${ }^{50}$.

J'ai suffisamment mis en relief, dans les pages précédentes, les incontestables qualités scientifiques du présent ouvrage pour qu'il soit superflu d'y revenir. Disons simplement pour conclure que l'évolution du Canada, de bien d'autres pays et de la société internationale en général donne à croire que le professeur Hugo Cyr - lui et ses distingués prédécesseurs ayant adopté la même thèse et qu'il n'oublie pas de citer — ont raison : les 
provinces canadiennes ont la compétence pour conclure et mettre en œuvre les traités sur les matières de leur ressort, et l'opinion des auteurs qui leur nient le pouvoir de conclure ces traités et souhaitent leur retirer celui de les mettre en œuvre législativement - opinion dont l'ouvrage n'omet jamais de faire état - a en 2012 quelque chose d'obsolète! 